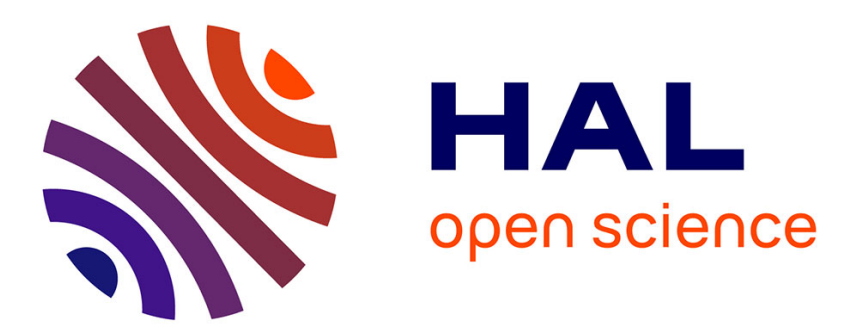

\title{
Electronic excitation of ethanol by low-energy electron impact
}

\author{
Alan Guilherme Falkowski, Marco Lima, Fábris Kossoski
}

\section{To cite this version:}

Alan Guilherme Falkowski, Marco Lima, Fábris Kossoski. Electronic excitation of ethanol by lowenergy electron impact. Journal of Chemical Physics, 2020, 152 (24), pp.244302. 10.1063/5.0008428 . hal-03518899

\section{HAL Id: hal-03518899 \\ https://hal.science/hal-03518899}

Submitted on 10 Jan 2022

HAL is a multi-disciplinary open access archive for the deposit and dissemination of scientific research documents, whether they are published or not. The documents may come from teaching and research institutions in France or abroad, or from public or private research centers.
L'archive ouverte pluridisciplinaire HAL, est destinée au dépôt et à la diffusion de documents scientifiques de niveau recherche, publiés ou non, émanant des établissements d'enseignement et de recherche français ou étrangers, des laboratoires publics ou privés. 


\title{
Electronic excitation of ethanol by low energy electron impact
}

\author{
Alan Guilherme Falkowski, ${ }^{1}$ Marco A. P. Lima, ${ }^{1}$ and Fábris Kossoski ${ }^{1}$, 2, a) \\ ${ }^{1)}$ Instituto de Física “Gleb Wataghin”, Universidade Estadual de Campinas, Campinas, \\ Brazil \\ ${ }^{2)}$ Aix Marseille University, CNRS, ICR, Marseille, France
}

(Dated: 10 January 2022)

We report computed differential cross sections (DCSs) for electron impact excitation of the lower lying states of both trans and gauche tautomers of ethanol, as well as total cross sections for the $15-50 \mathrm{eV}$ energy range. The Schwinger multichannel (SMC) method with pseudopotentials has been employed, and in our most sophisticated calculation in terms of multichannel coupling, 431 open target states have been considered. We found overall good agreement to available experimental data at intermediate scattering angles and at higher impact energies. Although we have used a Born closure scheme for the higher partial waves, we have found discrepancies in the forward direction that were assigned to a poor description of the long-range component of the lower partial waves. Meanwhile, the lack of more Rydberg states could be related to the overestimated DCSs at lower energies. Missing open channels are usually evoked to explain the remaining discrepancies to experiment, but here we argue that other factors should also be involved. Aiming at an improved description of the target states, we have proposed a simple procedure for selecting the pairs of hole and particle orbitals, while keeping the single excitation prescription of the current SMC implementation. A quantitative assessment of the collision process should further consider the individual contribution of each tautomer, which presented quite distinct DCSs in some cases. Our computed excitation energies also support that the second absorption band of ethanol is comprised of three singlet states of each tautomer, rather than the previously suggested two or four states.

a)fabris.kossoski@univ-amu.fr 


\section{INTRODUCTION}

One of the 17 goals of the United Nations to achieve sustainable development is to provide affordable, reliable, sustainable and clean energy sources for all ${ }^{1}$. In order to promote this idea, nations have to develop new technologies, for making a more efficient use of the available energy sources. Ethanol is a renewable biofuel and a great candidate to partially replace fossil fuels. It can be produced from the sugarcane juices (rich in monosaccharides), or from other crops (such as corn), which release the alcohol when digested by yeasts. This process of ethanol production is know as first generation. Another way of producing this biofuel is by using the bagasse and straw of sugarcane as raw material. This strategy, know as second generation, requires a pre-treatment to separate the cellulose and hemicellulose from lignin, followed by an enzymatic process to produce additional sugar ${ }^{2}$. Recently, Amorim et al. ${ }^{3,4}$ proposed and experimentally demonstrated that the use of low cost chemical plasmas can break chemical bonds of the lignin material, thus facilitating the cellulose separation. Spyrou et al. ${ }^{5}$ used a low-temperature non-equilibrium plasma reactor in lignocellulosic material and verified that the plasma attacks the lignin bonds. On the theoretical side, Oliveira et al. ${ }^{6}$ have shown that the presence of anion shape resonances in the lignin subunits and its absence in the cellulose subunits could play a role in the experimental finding. Other processes for the production of second generation ethanol can be seen in Ref. 7.

For optimal use of ethanol as a biofuel, there is a need to improve the efficiency of engines, and theoretical simulations of the combustion chamber should help providing important insights. These simulations require collision cross sections for the different processes taking place among the constituents inside the engine environment ${ }^{8}$. In particular, low-energy electrons generated by the spark plug are constantly colliding with the gas phase ethanol molecules. Brunger reported in a review ${ }^{9}$ the importance of accurate cross sections in modelling transport of biofuels. He highlighted the need for elastic, excitation, fragmentation and ionization cross sections for electron-molecule collisions. Within this motivation, ionization cross sections for biofuels have been subject of a recent review ${ }^{10}$.

Khakoo et al. have reported a combined experimental and theoretical study on low-energy elastic electron scattering from methanol and ethanol molecules ${ }^{11}$. Elastic cross sections have also

been obtained with the R-matrix calculations of Fujimoto et al. ${ }^{12}$ Studies on dissociative electron attachment to ethanol include those of Prabhudesai et al. ${ }^{13}$, Orzol et al. ${ }^{14}$ and Ibănescu et al. ${ }^{15}$ The last one also reported vibrational excitation cross sections. At intermediate energies, experi- 
mental and/or theoretical elastic and total cross sections have also been presented ${ }^{16-20}$. Recently, Sunanda et al. reported an experimental and theoretical study about the electronically excited states of ethanol ${ }^{21}$. There is only one paper about electronic excitation of ethanol by low energy electron collisions, by Hargreaves et al. ${ }^{22}$ However, the comparison between measurements and calculations falls short and the origin of the observed discrepancies is not clear.

In view of the need for reliable electron scattering cross sections and the poor agreement (compared to measurements) of the previous calculation for electronic excitation ${ }^{22}$, we decided to carry out a new theoretical investigation about the collision of low-energy electrons with ethanol molecules. The scattering calculations were performed with the Schwinger Multichannel method $(\mathrm{SMC})^{23-26}$ implemented with pseudopotentials ${ }^{27}$. Despite making use of the same method as the previous work ${ }^{22}$, our calculations differ from it in three fundamental aspects: (i) it considers both trans and gauche tautomers of ethanol, while only the former was taken into account before, (ii) it provides a more accurate description of the excited states, and (iii) it accounts for the presence of substantially more open channels. Here we address whether these aspects improve the quality of the computed electronic excitation differential cross sections (DCSs), by comparing our results to the previous calculation and to the available measured data ${ }^{22}$. In addition, total cross sections (TCSs) between 15 and $50 \mathrm{eV}$ are also reported and compared to previous results ${ }^{16-20}$.

\section{THEORY AND COMPUTATIONAL ASPECTS}

Since a review of the SMC method can be found elsewhere ${ }^{26}$, here we only summarize some of its key features, while we focus on the details of the present applications. The working expression for the fixed nuclei body-frame scattering amplitude is given by:

$$
f\left(\mathbf{k}_{\mathbf{f}}, \mathbf{k}_{\mathbf{i}}\right)=-\frac{1}{2 \pi} \sum_{m, n}\left\langle S_{\mathbf{k}_{\mathbf{f}}}|V| \chi_{m}\right\rangle\left(d^{-1}\right)_{m n}\left\langle\chi_{n}|V| S_{\mathbf{k}_{\mathbf{i}}}\right\rangle,
$$

where

$$
d_{m n}=\left\langle\chi_{m}\left|\left[\frac{\hat{H}}{N+1}-\frac{\hat{H} P+P \hat{H}}{2}+\frac{V P+P V}{2}-V G_{P}^{(+)} V\right]\right| \chi_{n}\right\rangle .
$$

$V$ is the interaction potential between the incident electron and the molecule, $\mathbf{k}_{\mathbf{i}}\left(\mathbf{k}_{\mathbf{f}}\right)$ is the incoming (outgoing) projectile wave vector, $\hat{H}=E-H$ is the total energy (target ground state energy plus the kinetic energy of the incoming electron) minus the $(N+1)$-electron Hamiltonian. The latter is given by $H=H_{0}+V$, where $H_{0}$ describes the non-interacting electron-molecule system and $\left|S_{\mathbf{k}_{\mathbf{i}}}\right\rangle$ is a solution of $H_{0}$, given by the product of a plane wave with momentum $\mathbf{k}_{\mathbf{i}}$ and a target 
state $\left|\Phi_{i}\right\rangle . G_{P}^{( \pm)}$is the Green's function operator projected onto the $P$ space, defined by the $N_{\text {open }}$ energetically open target electronic states $\left(P=\sum_{i=1}^{N_{\text {open }}}\left|\Phi_{i}\right\rangle\left\langle\Phi_{i}\right|\right)$. These are expanded in a set of singly-excited spin-adapted Slater determinants $\left|\phi_{r}^{S}\right\rangle$, whose selection is discussed below. Here, $r$ defines the pair of hole and particle orbitals, and $S$ denotes the spin coupling of this single excitation ( $S=0$ for singlets and $S=1$ for triplets). For the expansion of the variational scattering wave function, the method employs trial basis sets comprising $(N+1)$ - particle configuration state functions (CSFs), denoted by $\left|\chi_{m}\right\rangle$, that are built from spin-adapted, anti-symmetrized products of single excitations of the target and projectile scattering orbitals. The CSFs are given by $\left|\chi_{m}\right\rangle=$ $\mathscr{A}_{N+1}\left[\left|\phi_{r}^{S}\right\rangle \otimes\left|\varphi_{j}\right\rangle\right]$, where $r=0$ corresponds to the ground state and $r>0$ to singlet or triplet singly excited determinants, obtained by promoting one electron from a hole orbital (occupied for $r=0$ ) into a particle orbital (unoccupied for $r=0$ ). Both particle orbitals and the scattering orbitals $\left|\varphi_{j}\right\rangle$ are represented by the virtual orbitals that stem from the target ground state calculation. Finally, only doublet CSFs are retained in the scattering wavefunction expansion.

In applications of the SMC method, the excited states are usually described within the socalled MOB-SCI strategy (minimal orbital basis for single configuration interaction) ${ }^{28}$. As the name suggests, the idea is to perform a configuration interaction with single excitations (CIS), but restricted to a selected number of spin-adapted excitations from hole to particle orbitals (the minimal orbital basis). The pairs are manually included in the calculation until the excited states of interest are qualitatively described, which is ensured by comparing the MOB-SCI results to CIS calculations that employ all single excitations (full-CIS, or FCIS). The main motivation for the MOB-SCI strategy was to describe singlets and triplets states at the same time, since the improved virtual orbitals (IVOs) ${ }^{29}$ in the original implementation of the SMC method ${ }^{23,24}$ allowed only one of them to be well described. By mixing singlet and triplet IVOs, we constructed an orbital basis such that a single excitation configuration interaction technique allowed states of both multiplicities to be equally described.

Here we introduce a new procedure for selecting the excitations $\left|\phi_{r}^{S}\right\rangle$ to be included in the truncated CIS (TCIS) description of the target states. The key idea is to keep the excitations that present the larger overall coefficients of the FCIS calculation, for a given energy interval. Initially, the excited states are expanded in the set of all $\left|\phi_{r}^{S}\right\rangle$ determinants (a FCIS expansion):

$$
\left|\Phi_{i}^{S}\right\rangle^{\mathrm{FCIS}}=\sum_{r=1}^{N} c_{r, i}^{S}\left|\phi_{r}^{S}\right\rangle
$$

where the state index $i$ runs over both spin states. Then, a maximum number of excited states $N_{\text {open }}$ 
(including both multiplicities) is chosen (or, equivalently, a cutoff energy $\varepsilon_{\text {open }}$ ), which defines the weights:

$$
P_{r}^{N_{\text {open }}}=\sum_{i=1}^{N_{\text {open }}} \sum_{S=0}^{1}\left|c_{r, i}^{S}\right|^{2} .
$$

These are listed in decreasing importance: $P_{r_{1}}^{N_{\text {open }}}>P_{r_{2}}^{N_{\text {open }}}>\ldots>P_{r_{N}}^{N_{\text {open }}}$, and the $\left|\phi_{r}^{S}\right\rangle$ excitations associated to the $N_{\text {pairs }}$ first entries of the list (from $r_{1}$ up to $r_{N_{\text {pairs }}}$ ) are selected for the TCIS expansion that is effectively employed to describe the excited states in the scattering calculations:

$$
\left|\Phi_{i}^{S}\right\rangle^{\mathrm{TCIS}}=\sum_{j=1}^{N_{\text {pairs }}} d_{r_{j}, i}^{S}\left|\phi_{r_{j}}^{S}\right\rangle .
$$

In usual applications of the MOB-SCI approach, a limited set of excitations (5-50) are manually selected for the description of the 10-100 lower-lying excited states. ${ }^{30-38}$ Here, counting with recent improvements on the computational code and the proposed criterion based on the FCIS weights, an extended set of excitations (up to $\approx 250$ ) is automatically selected, which allows for up to $\approx 500$ excited states to be accounted for in the multichannel scattering calculations. The cutoff energy $\varepsilon_{\text {open }}$ and the number of hole-particle pairs $N_{\text {pairs }}$ are the two parameters that define the quality of the TCIS expansion (Eq. 5).

Ethanol can be found in three tautomers ${ }^{39-41}$, which are depicted in Fig. 1. The trans tautomer (also called anti) has a plane of symmetry (HOCC dihedral angle is $180^{\circ}$ ) and is slightly more stable than the degenerate gauche+ and gauche- tautomers, where the HOCC dihedral angle lies around $60^{\circ}$. Here we will simply refer to the gauche tautomer, since both gauche+ and gaucheshare the same chemical properties. Under gas-phase conditions, and at room temperature, around $61 \%$ of the ethanol molecules are found in the gauche configuration, and $39 \%$ in the trans ${ }^{39-41}$. The geometries of both tautomers were optimized with the second order perturbation theory of Moller-Plesset $^{42}$, using the aug-cc-pVDZ ${ }^{43}$ basis set, as implemented in the GAMESS package. The electronic ground state was described at the restricted Hartree-Fock approximation. Nuclei and the core electrons of carbon and oxygen atoms were represented by pseudopotentials ${ }^{27,44}$, while Cartesian Gaussian functions adapted to these pseudopotentials ${ }^{45}$ were used to describe the valence electrons. The same atomic basis set used in Ref. 6 was adopted here, which comprises a $5 s 5 p 2 d$ set for the carbon and oxygen atoms and a $3 s$ set for the hydrogen atoms. This set was supplemented with one $s$ function with exponent 0.009996 (0.016301), one $p$ with 0.024083 (0.013053) and one $d$ with 0.039188 (0.045190), centered at the carbon (oxygen) atoms. One $p$ function (exponent 0.75 ) was further added to the hydrogen atoms, which adds up to a total of 
162 Cartesian Gaussian functions. In order to have a more compact description of the excited states, we employed IVOs to represent both particle and scattering orbitals. They were generated by considering all singlet-coupled single excitations from the highest occupied molecular orbital to all canonical virtual orbitals.
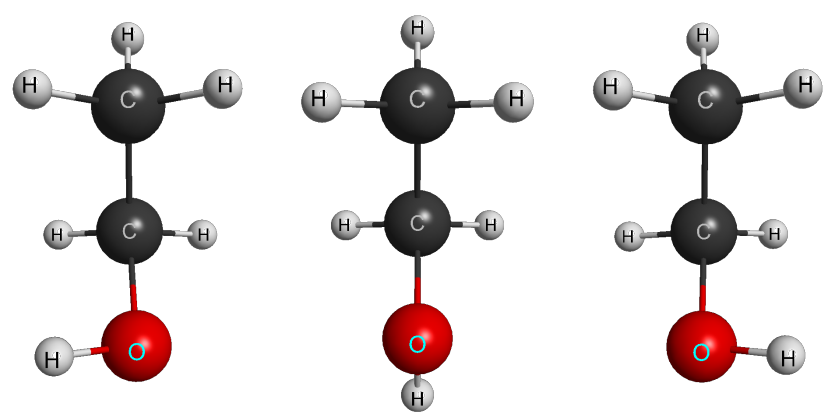

FIG. 1. Conformations of the ethanol molecule $\left(\mathrm{C}_{2} \mathrm{H}_{5} \mathrm{OH}\right)$ : gauche+ (left), trans (center) and gauche(right).

In the present study, the cutoff energy $\varepsilon_{\text {open }}$ was chosen to coincide with each impact energy of interest. Then, we selected the largest $N_{\text {pairs }}$ such that all the $N_{\text {open }}$ excited states lied below the cutoff energy $\varepsilon_{\text {open }}$. Finally, the CSF space was built with the same set of $N_{\text {pairs }}$ single excitations, while all the virtual orbitals were employed as scattering orbitals. This protocol guarantees that each target state below a given impact energy will be included in the scattering calculations as an open channel, within the limitations of the TCIS description. On the other hand, it also implies that the target states and the CSF space will be described at different levels for each energy. For the trans tautomer, calculations were performed for $\varepsilon_{\text {open }}=10.5 \mathrm{eV}, 12 \mathrm{eV}, 15 \mathrm{eV}, 17.5 \mathrm{eV}$ and $20 \mathrm{eV}$, which corresponded to $N_{\text {open }}=11,41,183,317$ and 431 open channels. Due to the more computationally demanding calculations for the gauche tautomer (due to its lack of symmetry), we only considered $N_{\text {open }}=11,41$ and 183 . We have not discarded combinations of CSFs via singular value decomposition of the denominator matrix (Eq. 2). Finally, the conformational average was computed based on the previously reported populations at room temperature $(61 \%$ for gauche and $39 \%$ for trans) $)^{39-41}$.

The use of square-integrable functions $\left(L^{2}\right)$ only to describe the scattering wavefunction effectively truncates the range of the interaction potential in the SMC method. Therefore, the long range dipole potential (and dipole allowed transitions) are not fully described, which compromises the computed DCSs at small scattering angles. We circumvented this issue with the well-known 
Born-Closure procedure ${ }^{46}$, where the partial scattering amplitudes up to $l_{\text {max }}$ are described with the SMC method $f_{l m}^{\mathrm{SMC}}$, while the higher ones are obtained via the first Born approximation (FBA) to the dipole potential (transition dipole moment in the case of excitation) $f_{l m}^{\mathrm{FBA}}$. The Born-corrected scattering amplitude in the lab frame then becomes:

$$
f_{\text {closure }}\left(\mathbf{k}_{\mathbf{f}}, \mathbf{k}_{\mathbf{i}}\right)=f^{\mathrm{FBA}}\left(\mathbf{k}_{\mathbf{f}}, \mathbf{k}_{\mathbf{i}}\right)+\sum_{l=0}^{l_{\max }} \sum_{m=-l}^{l}\left[f_{l m}^{\mathrm{SMC}}\left(k_{f}, \mathbf{k}_{\mathbf{i}}\right)-f_{l m}^{\mathrm{FBA}}\left(k_{f}, \mathbf{k}_{\mathbf{i}}\right)\right] Y_{l m}\left(\hat{k}_{f}\right),
$$

where the decomposition into spherical harmonics $Y_{l m}\left(\hat{k}_{f}\right)$ is performed in terms of the scattered electron direction $\hat{k}_{f}$. We picked the smallest $l_{\max }$ that provided DCSs similar to those obtained with the SMC calculation for intermediate scattering angles (larger than $\approx 30^{\circ}$ ), where the long range component of the potential has a minor effect. While in the SMC calculations the excited states were described at the CIS level (as described above), the transition dipole moments employed in the Born-closure formula were obtained with the equation-of-motion coupled-cluster with singles and doubles (EOM-CCSD) ${ }^{47-49}$ level of theory and aug-cc-pVDZ basis set, as implemented in Gaussian ${ }^{50}$. An additional set of $1 s 1 p 1 d$ diffuse functions was placed at the carbon and oxygen atoms, with exponents obtained by dividing the last exponents of the aug-cc-pVDZ basis set by 4 . The excitations energies obtained with the EOM-CCSD calculations have also led us to a new interpretation of the second band of excited states of ethanol.

\section{RESULTS AND DISCUSSION}

In Fig. 2 we present elastic DCSs, computed for the trans tautomer and for the Boltzmann average of the trans and gauche tautomers. In all DCSs here presented, the experimental data obtained at $10 \mathrm{eV}$ were compared to calculations performed at $10.5 \mathrm{eV}$. We have not performed the Born-closure correction to the elastic channel, since that should affect the DCSs only at the very small scattering angles (below $\left.\approx 15^{\circ}\right)^{11}$. As we account for more open channel in our calculations, the magnitudes of the DCSs decrease above $\approx 30^{\circ}$, since the elastic channel now competes with the electronic excitation channels for the probability flux, which has been extensively demonstrated $^{30-38}$. While the qualitative behaviour of the trans and gauche elastic DCSs is rather similar, there are systematic differences in their magnitudes. The DCSs of the gauche tautomer have a smaller magnitude at forward (below $\approx 40^{\circ}$ ) and backward (above $\approx 130^{\circ}$ ) scattering angles, and a larger magnitude at the intermediate angles, when compared to the trans tautomer. This effect is particularly noticeable above $17.5 \mathrm{eV}$, where the minimum of the DCS at $90-120^{\circ}$ becomes less 
pronounced when accounting for both tautomers.
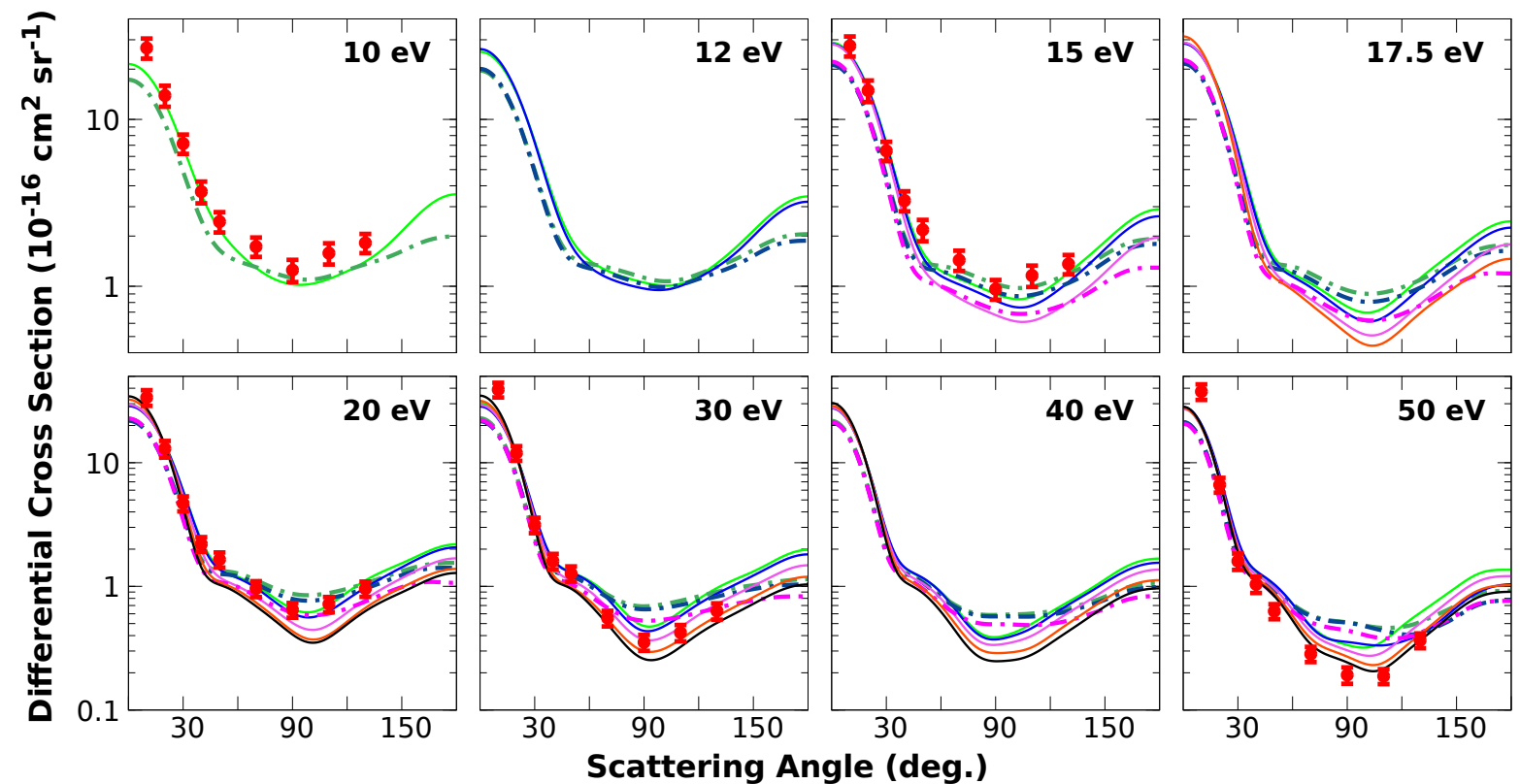

FIG. 2. Elastic differential cross sections. Full lines correspond to the results for the trans tautomer, and dot-dashed lines to the room temperature trans+gauche average. The following numbers of open channels were considered in the calculations: 11 (green), 41 (blue), 183 (magenta), 317 (orange) and 431 (black). The red dots are the experimental results of Khakoo et $\mathrm{l}^{11}$.

The comparison to the available experimental data ${ }^{11}$ reveals important aspects about the approximations employed in our scattering calculations. Surprisingly, the computed elastic DCSs often lies below the experimental curves. This is in contrast to previous multichannel calculations with the SMC method, where theory either overestimates or presents good agreement to experimental elastic DCSs ${ }^{30-32}$. Including more open channels improved agreement to experiment in these previous reports, and thus the remaining discrepancies were always assigned to the limited number of open channels. In the present case, however, including even more open channels would further decrease the DCSs, hence worsening the comparison. Therefore, it seems that augmenting the open channel space is not enough for achieving accurate cross sections, as the present results indicate that other factors also play a role. It is possible that multichannel coupling effects associated to higher angular momenta are not well described in our calculations, due to the difficulties of the SMC method in accounting for long-range interactions. Inclusion of diffuse basis functions at extra centers should improve the representation of both lower and higher partial waves, and we plan to investigate this aspect in future studies. Another possibility is that the competition for the 
probability flux is particularly sensitive to the level of description of the target states, and if that is the case we would have to go beyond the current Hartree-Fock description for the ground state and CIS prescription for the electronic excited states.

A quantitative comparison to experiment should also account for the presence of both ethanol tautomers. For example, agreement at $20 \mathrm{eV}$ for the intermediate angles seems to improve when both forms are considered. On the other hand, not doing so would misleadingly indicate a rather good agreement with experiment below $\approx 30^{\circ}$. Previous elastic scattering calculations (also presented in Ref. 11) had good agreement with the measurements below $20 \mathrm{eV}$. However, we believe this would be somewhat fortuitous, since the calculations were performed only for the trans tautomer, and considering only the elastic channel as open.

Before presenting the DCSs for electronic excitation, we are first going to discuss the assignments of the lower-lying excited states in light of our EOM-CCSD results. The excitation energies for the 10 first excited states of both tautomers are summarized in Table I, as obtained from our calculations and from previous reports ${ }^{21,22}$. Ethanol has a weak absorption band at $6-7.5 \mathrm{eV}$, and a more intense one in the $7.5-8.5 \mathrm{eV}$ region ${ }^{21,51-54}$. The first band stems exclusively from the $\mathrm{S}_{1}$ state, where an electron is excited from the HOMO (mostly $n\left(a^{\prime \prime}\right)$ out-of-plane oxygen lone pair orbital) to an orbital with mixed $\sigma_{\mathrm{OH}}^{*}$ valence and $3 s$ Rydberg character ${ }^{21,51-54}$. Our computed oscillator strengths are 4 times larger for the gauche tautomer (0.0116) than for the trans tautomer (0.0030), indicating that the former should contribute the most for the first band. The computed excitation energy of $6.75 \mathrm{eV}$ matches very well with what has been recently obtained in high-resolution VUV photoabsorption spectra measurements $(6.77 \mathrm{eV})^{21}$, and also with timedependent density functional theory (TDDFT) calculations $(6.58 \mathrm{eV})^{21}$.

However, the interpretation of the second band is not yet settled. Previous studies ${ }^{51-54}$ suggested that $\mathrm{S}_{2}$ and $\mathrm{S}_{3}$ states should be involved, which have $n\left(a^{\prime \prime}\right) \rightarrow 3 p\left(a^{\prime \prime}\right)$ and $n\left(a^{\prime \prime}\right) \rightarrow 3 p\left(a^{\prime}\right)$ Rydberg character. But the aforementioned VUV measurements (supported by TDDFT calculations $)^{21}$ support that the additional $n\left(a^{\prime \prime}\right) \rightarrow 3 p\left(a^{\prime}\right)$ and $n\left(a^{\prime}\right) \rightarrow \sigma_{\mathrm{OH}}^{*} / 3 s\left(\mathrm{~S}_{4}\right.$ and $\left.\mathrm{S}_{5}\right)$ Rydberg states would also participate to the second band. When compared to the VUV assignments there is excellent agreement for the excitation energies of the $3 p$ Rydberg states, for both EOM-CCSD and TDDFT calculations $^{21}$, with an average difference of only $0.1 \mathrm{eV}$. Thus, our results also support the involvement of $S_{2}, S_{3}$ and $S_{4}$ states. On the other hand, the EOM-CCSD calculations indicate the $\mathrm{S}_{5}$ state (of both tautomers) would be $0.4 \mathrm{eV}$ above the previous results ${ }^{21}$, thus beyond the band range. Therefore, we believe the $7.5-8.5 \mathrm{eV}$ band in ethanol would not be comprised of 
TABLE I. Energies (in eV) for the 10 first excited states of both trans and gauche tautomers, according to our EOM-CCSD calculations, and to previously reported TDDFT $^{21}$ calculations and VUV ${ }^{21}$ and EELS ${ }^{22}$ measurements.

\begin{tabular}{|c|c|c|c|c|c|c|c|c|}
\hline & \multicolumn{4}{|c|}{ Trans } & \multicolumn{4}{|c|}{ Gauche } \\
\hline State & EOM-CCSD & $\mathrm{TDDFT}^{21}$ & $\mathrm{VUV}^{21}$ & EELS $^{22}$ & EOM-CCSD & $\mathrm{TDDFT}^{21}$ & $\mathrm{VUV}^{21}$ & EELS $^{22}$ \\
\hline $\mathrm{T}_{1}$ & 6.37 & 6.52 & & 6.27 & 6.51 & & & 6.27 \\
\hline $\mathrm{S}_{1}$ & 6.60 & 6.46 & 6.77 & 6.65 & 6.75 & 6.58 & 6.77 & 6.65 \\
\hline $\mathrm{T}_{2}$ & 7.66 & 7.56 & & 7.63 & 7.69 & & & 7.63 \\
\hline $\mathrm{S}_{2}$ & 7.81 & 7.51 & 7.61 & 7.71 & 7.83 & 7.59 & 7.70 & 7.71 \\
\hline $\mathrm{T}_{3}$ & 7.88 & 7.89 & & & 7.98 & & & \\
\hline $\mathrm{S}_{3}$ & 7.99 & 7.83 & 7.91 & & 8.10 & 7.89 & 7.97 & \\
\hline $\mathrm{T}_{4}$ & 8.02 & 7.93 & & & 8.06 & & & \\
\hline $\mathrm{S}_{4}$ & 8.07 & 7.89 & 8.08 & & 8.13 & 7.96 & 8.14 & \\
\hline $\mathrm{T}_{5}$ & 8.37 & 8.33 & & & 8.32 & & & \\
\hline $\mathrm{S}_{5}$ & 8.64 & 8.28 & 8.24 & & 8.56 & 8.12 & 8.18 & \\
\hline
\end{tabular}

two ${ }^{51-54}$ or four ${ }^{21}$ excited states (from each tautomer), but three instead $\left(\mathrm{S}_{2}, \mathrm{~S}_{3}\right.$ and $\left.\mathrm{S}_{4}\right)$.

Our computed DCSs are going to be compared to those reported by Hargreaves et al. ${ }^{22}$, which were obtained by electron energy loss spectra (EELS) measurements. In these experiments, excitations to triplet states can take place, which is not the case in VUV absorption. According to our calculations, the lower-lying triplets appear $0.1-0.25 \mathrm{eV}$ below the singlet states, such that each pair would contribute to the same observed band. In their fitting procedure of the measured EELS, the authors have referenced to the interpretation presented in the older papers ${ }^{51-54}$. Thus, their interpretation of the second band would be compromised. In light of the above discussion, three states of each multiplicity should contribute to the DCSs that have been assigned to this band ${ }^{22}$, and our later comparison to the calculations is performed accordingly.

In our scattering calculations, the excited states had to be described with a CIS wavefunction, in view of the current implementation of the SMC method. The energies computed for the 8 first excited states of the trans tautomer are presented in Table II, as obtained from the TCIS calculations for $N_{\text {pairs }}=5,20,91,158$ and 215 pairs, and also from the FCIS calculations (1520 pairs). In the previous theoretical study about electron impact excitation of ethanol ${ }^{22}$, the authors 
have described each excited state with one single excitation, and their reported energies lied in average $0.17 \mathrm{eV}$ above what we found in our crudest calculation, with $N_{\text {pairs }}=5$. The excited states are further stabilized by an average of $0.26 \mathrm{eV}$ in our most sophisticated calculation $\left(N_{\text {pairs }}=215\right)$, thus getting only $0.15 \mathrm{eV}$ (in average) above the FCIS result. In addition, Fig. 3 illustrates how the number of states and the energy levels behave as the number of single excitations is increased, as selected according to the criterion we have presented in the previous section. In particular, there are 431 states below $\varepsilon_{\text {open }}=20 \mathrm{eV}$ in the $N_{\text {pairs }}=215$ calculation, which is close to the set of 545 states (below $20 \mathrm{eV}$ ) described at the FCIS level. Single excitations still provide a limited representation of the excited states, and even the FCIS calculation would overestimate their real energies by $\approx 1.5 \mathrm{eV}$, as can be seen by comparing Tab. I and Tab. II.

TABLE II. Energies (in eV) for the 8 first excited states of the trans tautomer, according to our CIS calculations for $N_{\text {pairs }}=5,20,91,158$ and 215 (which were employed for the scattering calculations with $N_{\text {open }}=11,41,183,317$ and 431, respectively), to our FCIS result (1520 pairs) and to the singly-excited CI calculation with improved virtual orbital (IVO) employed in the previous scattering study ${ }^{22}$.

\begin{tabular}{c|ccccccc}
\hline \hline State & 5 & 20 & 91 & 158 & 215 & FCIS & IVO $^{22}$ \\
\hline $\mathrm{T}_{1}\left(1^{3} A^{\prime \prime}\right)$ & 8.12 & 8.13 & 7.90 & 7.80 & 7.78 & 7.53 & 8.28 \\
$\mathrm{~S}_{1}\left(1^{1} A^{\prime \prime}\right)$ & 8.37 & 8.34 & 8.18 & 8.11 & 8.09 & 8.00 & 8.54 \\
$\mathrm{~T}_{2}\left(2^{3} A^{\prime \prime}\right)$ & 9.33 & 9.35 & 9.25 & 9.12 & 9.09 & 8.86 & 9.42 \\
$\mathrm{~S}_{2}\left(2^{1} A^{\prime \prime}\right)$ & 9.56 & 9.51 & 9.43 & 9.36 & 9.35 & 9.29 & 9.69 \\
$\mathrm{~T}_{3}\left(1^{3} A^{\prime}\right)$ & 10.01 & 9.98 & 9.71 & 9.63 & 9.61 & 9.40 & 10.18 \\
$\mathrm{~S}_{3}\left(2^{1} A^{\prime}\right)$ & 9.71 & 9.67 & 9.65 & 9.63 & 9.62 & 9.57 & 10.02 \\
$\mathrm{~T}_{4}\left(3^{3} A^{\prime \prime}\right)$ & 9.70 & 9.70 & 9.67 & 9.61 & 9.60 & 9.40 & 10.10 \\
$\mathrm{~S}_{4}\left(3^{1} A^{\prime \prime}\right)$ & 9.77 & 9.76 & 9.73 & 9.69 & 9.68 & 9.65 & 10.48 \\
\hline \hline
\end{tabular}

The computed DCSs for the lowest lying excited state of ethanol are presented in Figs. 4, 5, 6 and 7. First, the general common features will be outlined, and then the particularities of each case will be discussed. As more excited states are included in the open channel space, the DCSs decrease in magnitude, in line with what has been observed in previous studies with the SMC method ${ }^{30-35,38}$. Inclusion of the lowest lying states has the greatest impact on the drop in magnitude, as seen in the shift from the $N_{\text {open }}=11$ to the $N_{\text {open }}=41$ calculations. As the higher lying states are accounted for, the effect on the computed DCSs becomes progressively smaller. 


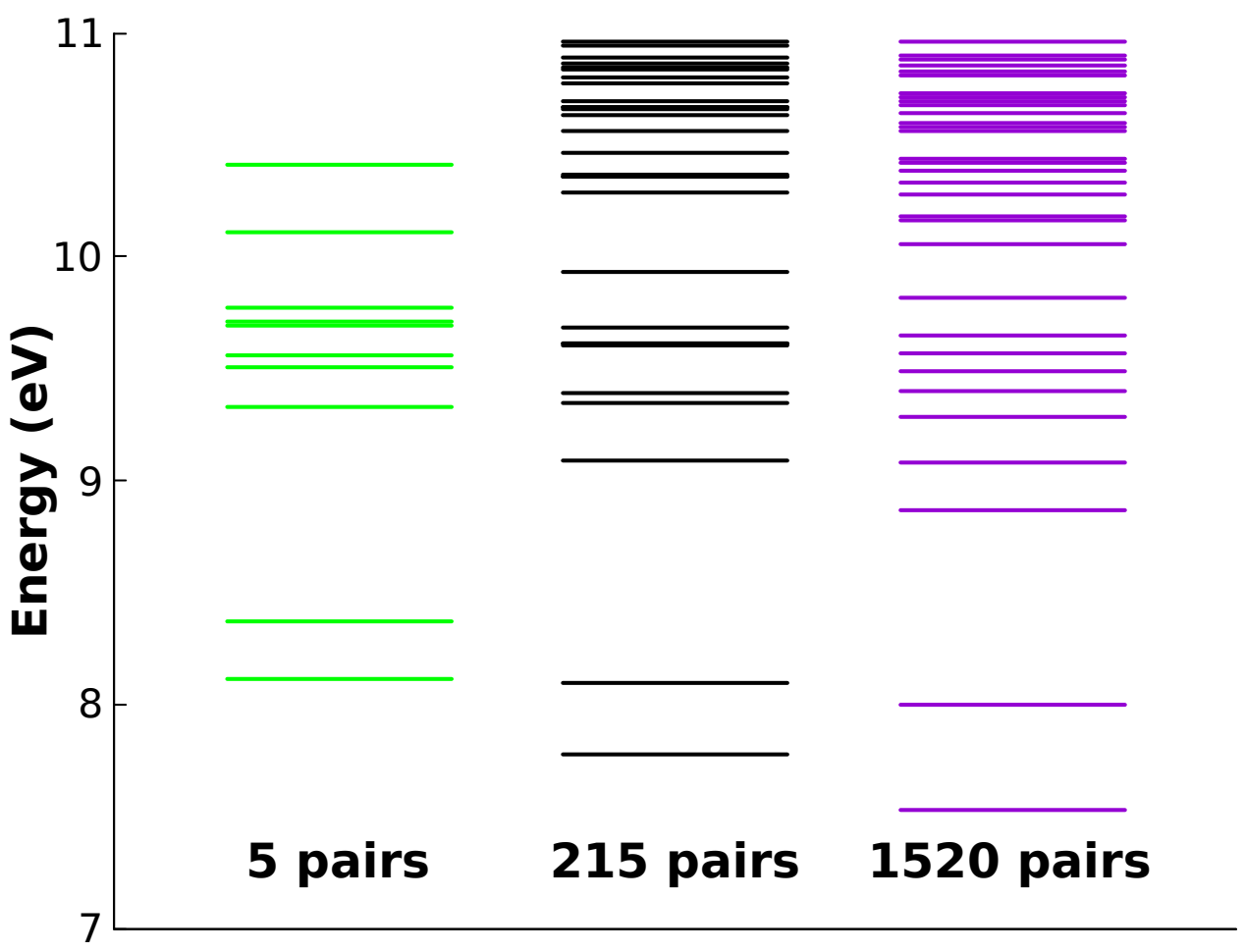

FIG. 3. Energy levels (up to to $11 \mathrm{eV}$ ) for the excited states of the trans tautomer of ethanol, computed for $\varepsilon_{\mathrm{open}}=20 \mathrm{eV}$ and $N_{\text {pairs }}=5,215$ and 1520 pairs (the last one corresponding to the FCIS result). The former two correspond to the $N_{\text {open }}=11$ and 431 scattering calculations.

At $20 \mathrm{eV}$, in particular, the magnitudes are relatively converged with respect to the number of open channels, for the chosen Gaussian Cartesian basis set and the employed TCIS expansion. This can be seen in the comparison between $N_{\text {open }}=431$ and $N_{\text {open }}=317$ results in Fig. 5, and even comparing to the $N_{\text {open }}=183$ case in Fig. 4 and Fig. 7. We also found that the shape of the trans and gauche curves are usually different, which makes the conformational averaged DCSs more isotropic. Additionally, there are relevant differences in their magnitudes (for the same level of calculation), and in most cases the gauche tautomer presented larger DCSs than the trans form.

Fig. 4 displays the DCSs for excitation of the $S_{1}$ state. Calculations provided systematically overestimated curves in comparison to the experimental data above $\approx 30^{\circ}$, but as more open channels are accounted for, the agreement becomes much better. At 15, 17.5 and $20 \mathrm{eV}$, the computed DCSs for the trans tautomer would lie below the experimental ones. On the other hand, the gauche tautomer presents larger magnitudes, such that the conformational average provides DCSs much closer to the experiment. The measured data display a strong rise in the forward direction, which is due to the long range dipole interaction, as the simpler FBA to the transition dipole moment 

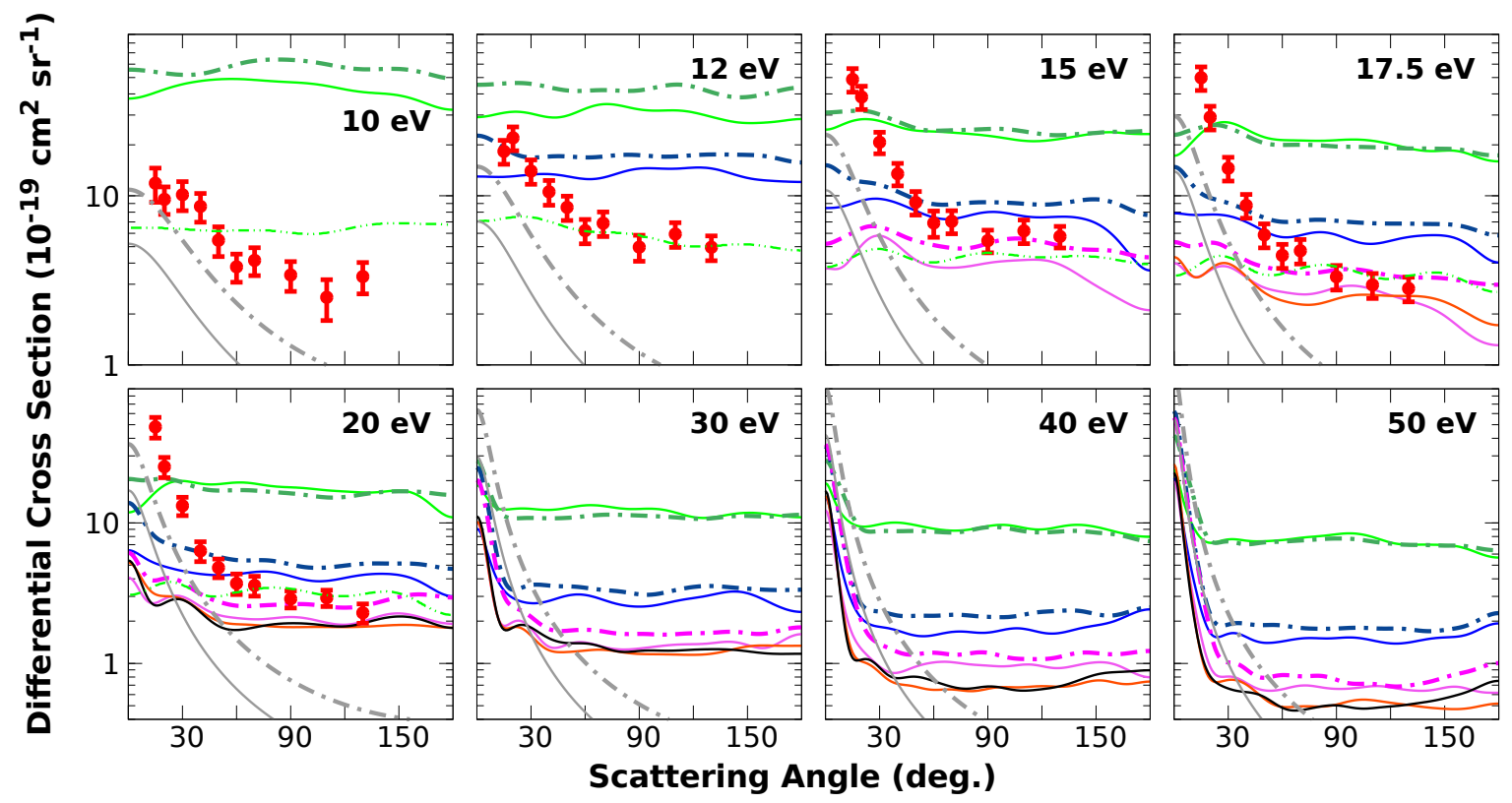

FIG. 4. Differential cross sections for electronic excitation of the $S_{1}$ state of ethanol. Full lines correspond to the results for the trans tautomer and dot-dashed lines to the room temperature trans+gauche average, for the following numbers of open channels in the calculations: 11 (green), 41 (blue), 183 (magenta), 317 (orange) and 431 (black). The grey lines correspond to the first Born approximation to the transition dipole moment. The dot-dot-dashed light green lines are from previous SMC calculations, and the red dots are the experimental results, both reported in Ref. 22.

is able to qualitatively reproduce it. As expected, the forward peak becomes increasingly more pronounced as the impact energy increases. But surprisingly, in the results corrected with the Born-closure procedure (Eq. 6), this feature is marked only at the higher energies. At lower energies, on the other hand, the Born correction has a rather small effect, as it only slightly increases the DCSs in the forward direction. Thus, in the lower energy regime, the higher partial waves are not really important in excitation induced by the long range dipole interaction. Both long-range interactions (which control the forward scattering) and short-range interactions (important at backward and intermediate angles) would be dominated by the lower partial waves. While the SMC calculations provide a fair description of the latter, it fails in the former. This probably reflects the lack of more diffuse functions of $s$ and $p$ type. In the FBA calculations, the magnitude of the forward peak is largely underestimated when only the trans tautomer is accounted for. The gauche tautomer has a relatively larger transition dipole moment $(0.26 \mathrm{D})$ than the trans $(0.13 \mathrm{D})$, which brings the conformational averaged DCSs closer to experiment at small angles. The remaining dif- 
ference might be related to the uncertainties in the calculation of the transition dipole moment and to the FBA itself. The worst agreement would be at $15 \mathrm{eV}$, where the FBA calculation provides DCSs below the experiment by a factor of $\approx 3$. Considering that the transition dipole moment could be underestimated by up to $40 \%$ (which is not uncommon), then the DCSs computed with the FBA would be underestimated by a factor close to 2 .
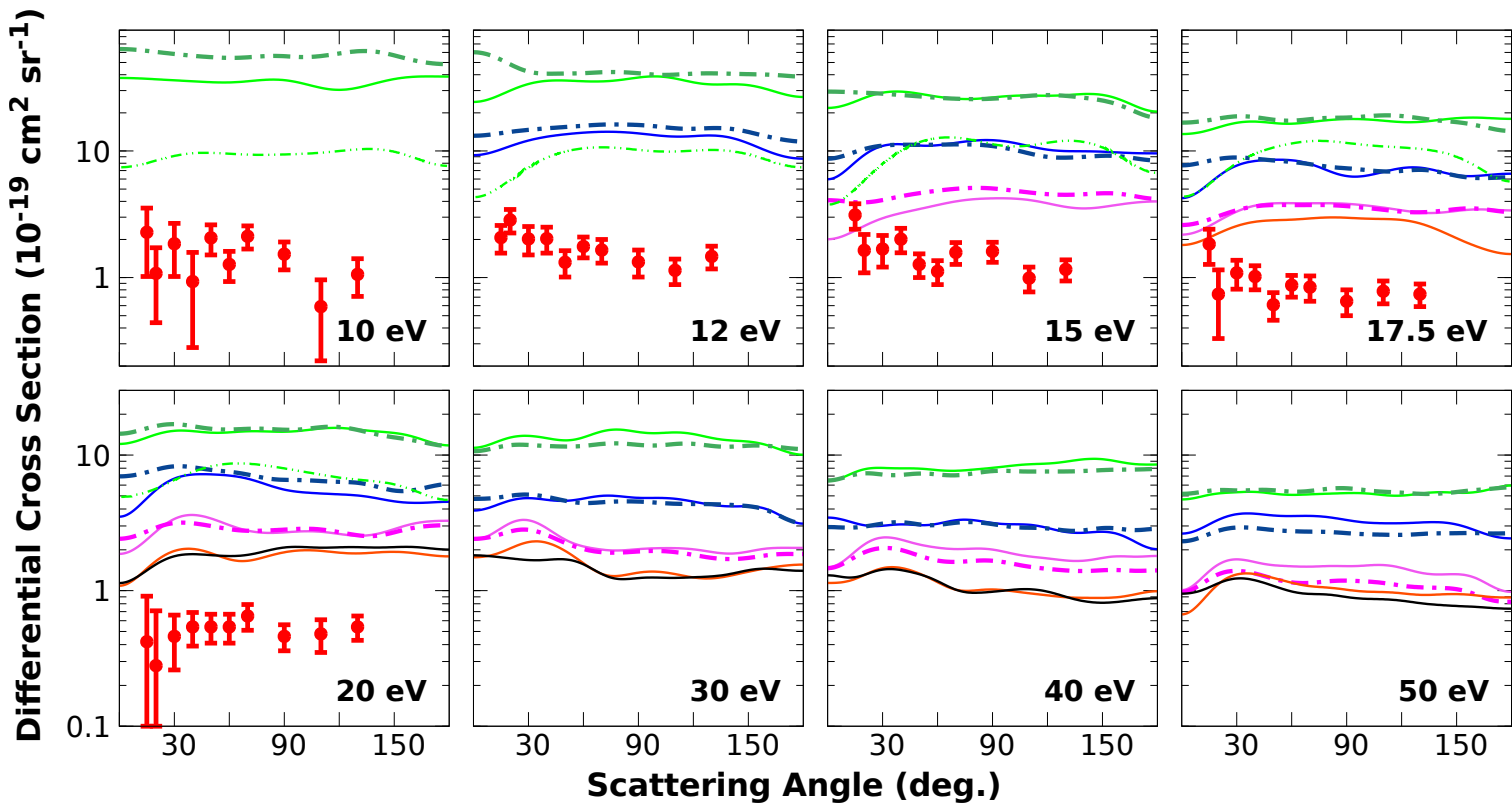

FIG. 5. As in Fig. 4, but for the $\mathrm{T}_{1}$ state.

The DCSs for the $T_{1}$ are presented in Fig. 5. Except for a mild forward scattering preference that was found experimentally at some energies (which we discuss in more detail later), both theory and experiment provided fairly isotropic DCSs. As in the case of $\mathrm{S}_{1}$, accounting for more open channels considerably improves agreement above $\approx 30^{\circ}$. However, magnitudes are still overestimated by a factor of 4 at $20 \mathrm{eV}$, and by a factor of 40 at $10 \mathrm{eV}$. As discussed in Section II, in our most sophisticated calculation at each impact energy, all excited states are considered to be open (given our choice of Gaussian basis set and CIS expansion). Thus, our results indicate that the including the missing excited states would still be required for further decreasing the magnitudes of the cross sections. Since agreement is worse at the lower energies, it seems that the major issue would be related to the lack of more Rydberg states, rather than the lack of doubly-excited states (which should become more important at higher energies). Recent photoabsorption measurements of ethanol have assigned around 15 singlet excited states (mostly Rydberg) below $10 \mathrm{eV}^{21}$, while only 5 singlet states could be described in our calculations (due to the lack of more diffuse basis 
functions and higher-order excitations). We believe that accounting for the missing Rydberg states should provide more quantitative agreement between calculated and experimental cross sections, specially at lower energies.
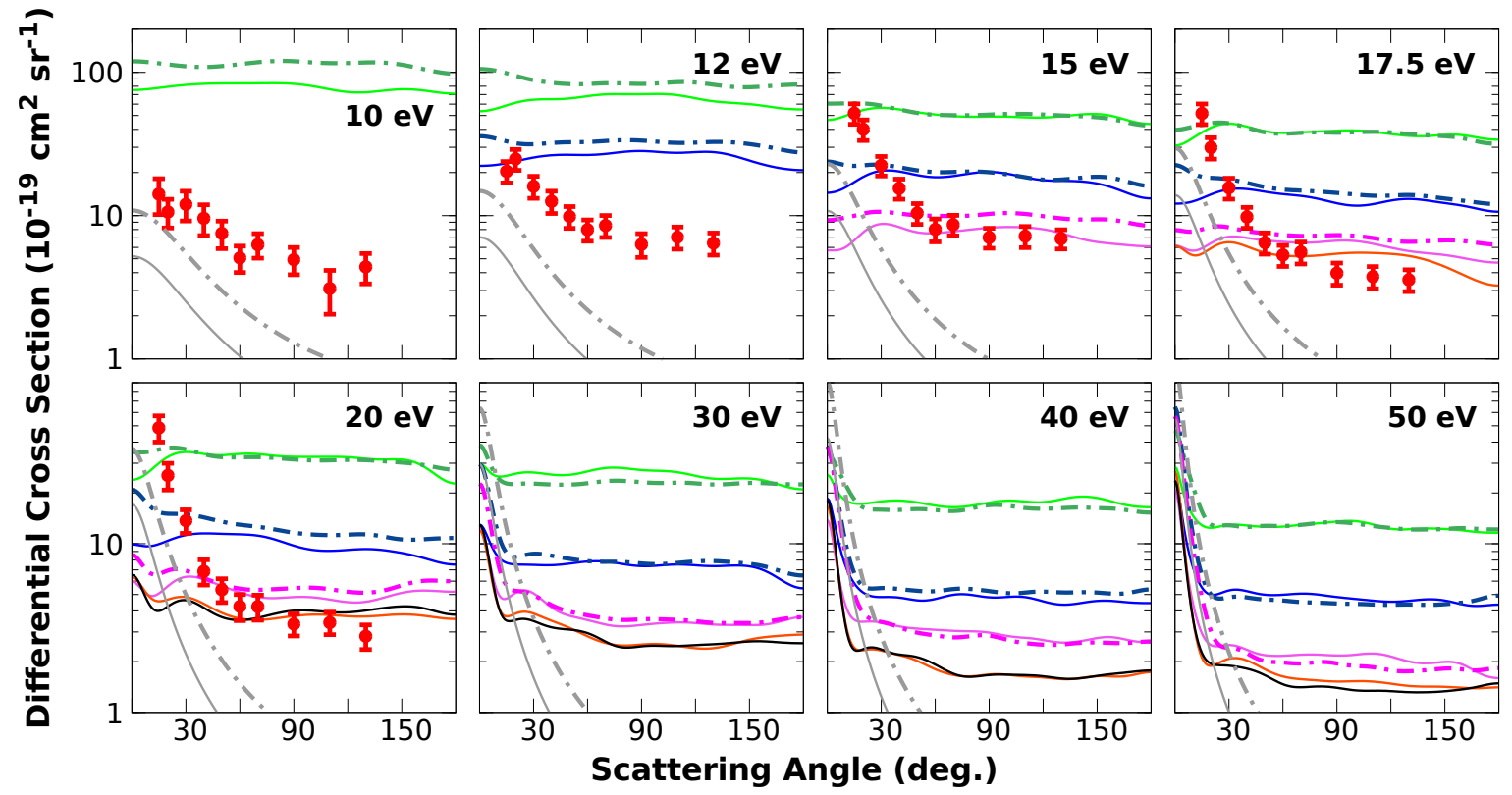

FIG. 6. As in Fig. 4, but for the summed contributions of the $\mathrm{T}_{1}$ and $\mathrm{S}_{1}$ states.

As discussed before, the first band of the measured EELS ${ }^{22}$ should encompass both $\mathrm{S}_{1}$ and $\mathrm{T}_{1}$ states. We believe that the procedure adopted in Ref. 22 could not fully unfold the contribution of each state, for the following reasons. First, the reported $\mathrm{T}_{1} \mathrm{DCS}$ s displayed an increase in the forward direction, which would not be expected for a triplet state, but rather for a singlet state. Second, the measured DCSs for the triplet states of the similar methanol molecule actually decrease in the forward direction ${ }^{55}$. And third, the $\mathrm{T}_{1} / \mathrm{S}_{1}$ energy gap is somewhat smaller according to our EOM-CCSD calculations $(0.23 \mathrm{eV})$ than what has been inferred from the EELS $(0.38 \mathrm{eV})^{22}$. Therefore, we also present the summed contribution of the DCSs for $S_{1}$ and $T_{1}$ states in Fig. 6. Taking the case of $20 \mathrm{eV}$, for instance, the computed DCSs (in the $N_{\text {open }}=431$ calculation) are underestimated for the $S_{1}$ state (Fig. 4), but overestimated for the $T_{1}$ state (Fig. 5), while there is a good matching for the summed contributions. Thus, at least part of the above discussed discrepancies for the individual $S_{1}$ and $T_{1}$ states could be related to the unfolding process of the measured EELS.

In view of our previous discussion about the distinct interpretations for the second band of ethanol and the inherent difficulties in separating the contribution of each state, we decided to 

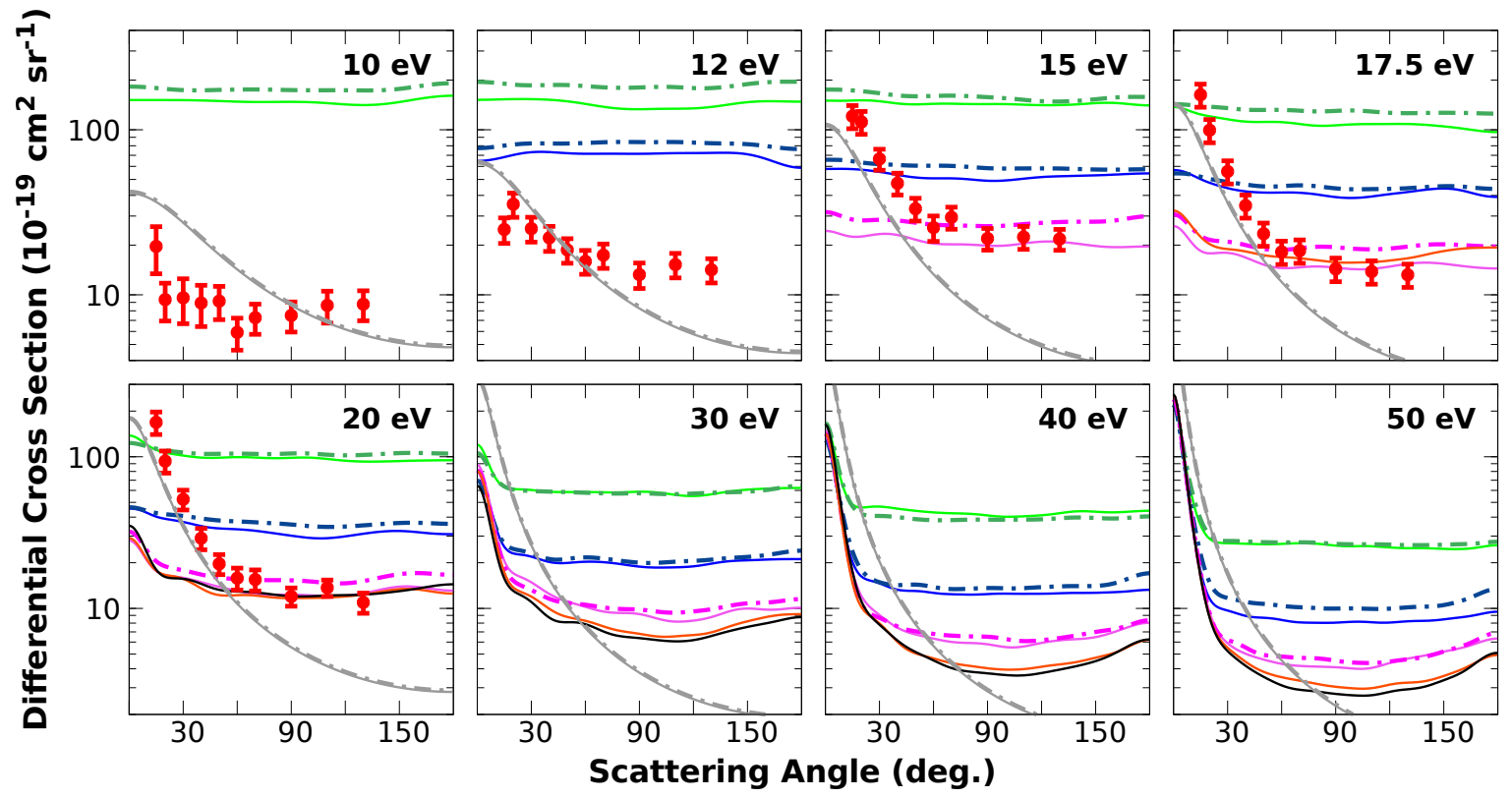

FIG. 7. As in Fig. 4, but for the summed contributions of the $T_{2}, T_{3}, T_{4}, S_{2}, S_{3}$ and $S_{4}$ states.

present in Fig. 7 the summed DCSs of the $T_{2}, T_{3}, T_{4}, S_{2}, S_{3}$ and $S_{4}$ states. They are compared to the sum of the measured triplet and singlet signals for this band ${ }^{22}$. The findings are similar to what we have already discussed. In our more sophisticated calculations, we are able to describe the shape and magnitude of the DCSs above $\approx 30^{\circ}$, at $15,17.5$ and $20 \mathrm{eV}$, while at lower energies the magnitudes are overestimated. Again, the FBA provides a fairly reasonable description of the forward scattering, but here both trans and gauche tautomers have comparable contributions. Importantly, our calculations reproduce the overall larger DCSs for excitation of the second band, when compared to the first one.

A lot of computational time was demanded in our most sophisticated calculations ( $N_{\text {open }}=$ 431), mostly due to the large CSF space it required. In order to achieve similar results with less computational effort, we have performed new rounds of scattering calculations with a reduced CSF space, while trying to preserve its most important CSFs. Instead of employing all $105 \mathrm{a}^{\prime}$ and $47 \mathrm{a}^{\prime \prime}$ virtual orbitals as scattering orbitals, we have selected the $68 \mathrm{a}^{\prime}$ and $30 \mathrm{a}^{\prime \prime}$ lower lying ones. With this smaller space, the extra calculations were performed for the trans tautomer, with $N_{\text {open }}=$ 11 and 431. In the latter case, the CSF space comprised a total of 47809 CSFs when all scattering orbitals are included, which is reduced to 29553 CSFs with the above selection. The results are compared in Fig. 8 for the first band and in Fig. 9 for the second one. The computed DCSs are consistently very close to each other, specially for $N_{\text {open }}=431$. The most time consuming stages 
of the SMC calculations scale with the squared number of CSFs, which implies in a factor of 2.6 in the present case. Our results show that strategies for reducing the CSF space can have a marginal effect on the computed cross sections, but a big one on the overall computational effort.
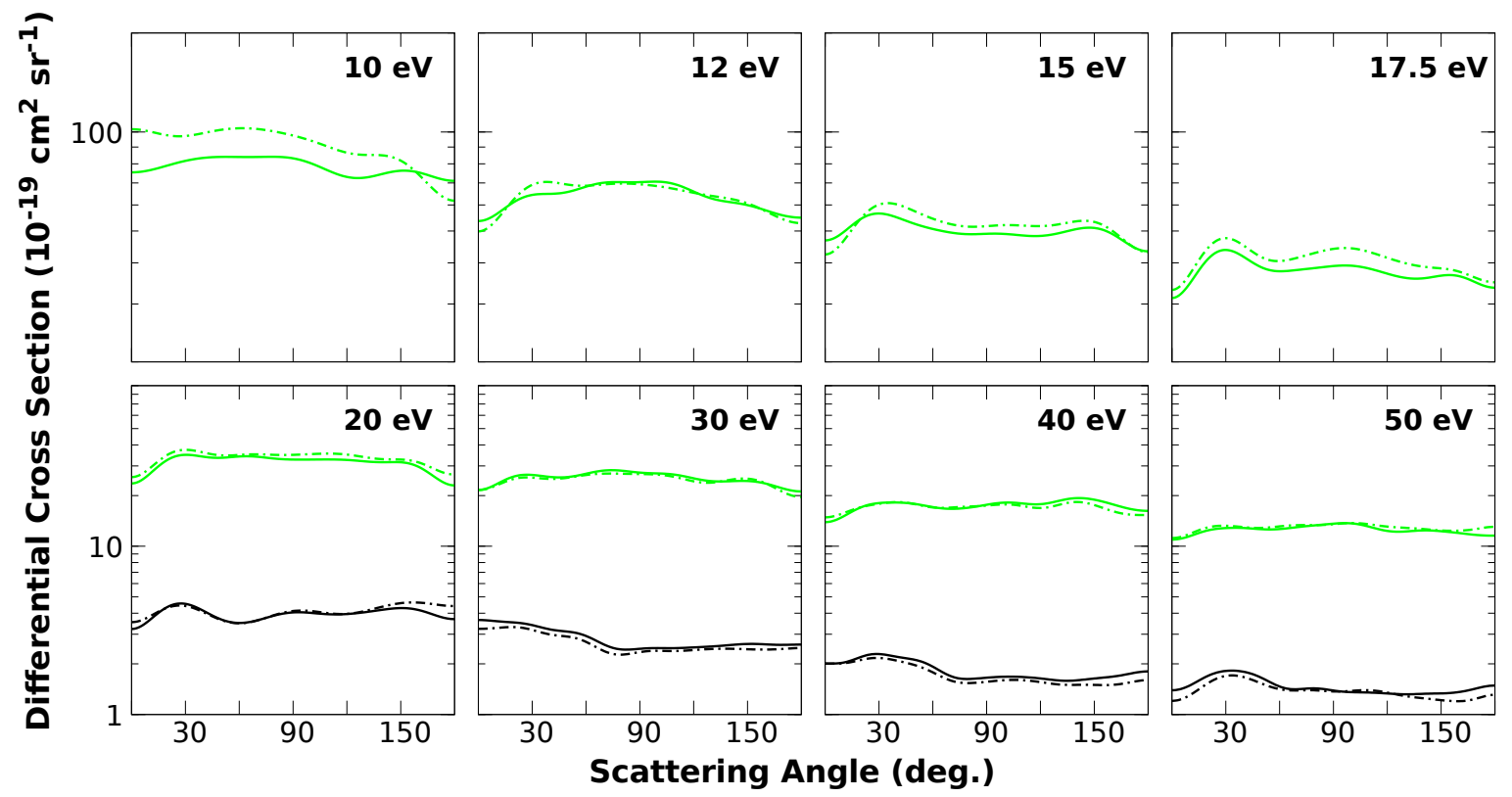

FIG. 8. Differential cross sections for electronic excitation of the first band (summed contributions of the $\mathrm{T}_{1}$ and $\mathrm{S}_{1}$ states) of the trans tautomer, according to the $N_{\mathrm{open}}=11$ (green) and 431 (black) calculations, without the Born-Closure correction. Full lines correspond to the calculations as described in Sec. II (all scattering orbitals), while dot-dashed lines correspond to the calculations as described in the end of Sec. III (selected scattering orbitals).

Finally, previous results ${ }^{16-20}$ for the TCSs are compared to our results in Fig. 10. The TCSs were computed in the $N_{\text {open }}=183$ calculation, which was the most sophisticated calculation that we managed to perform for both conformers. Results for other levels of multichannel coupling (not shown) are very similar, with differences on the magnitudes below $10 a_{0}^{2}$. The elastic components were corrected with the Born-closure procedure (dipole moments computed at the CCSD level), which provided a more or less systematic increase of $\approx 8 a_{0}^{2}$ to the TCSs. Fig. 10 shows that each conformer displays reasonably distinct TCSs. While the behaviour is the same, the magnitudes are larger for the trans conformer (by $22 a_{0}^{2}$ at $15 \mathrm{eV}$, slowly decreasing at higher impact energies). While elastic and electronic excitation channels are in principle described in the SMC calculations, ionization channels are missing in the method. In view of this limitation, we have also added to the SMC cross sections (magenta curves in Fig. 10) the ionization cross sections measured by Nixon 

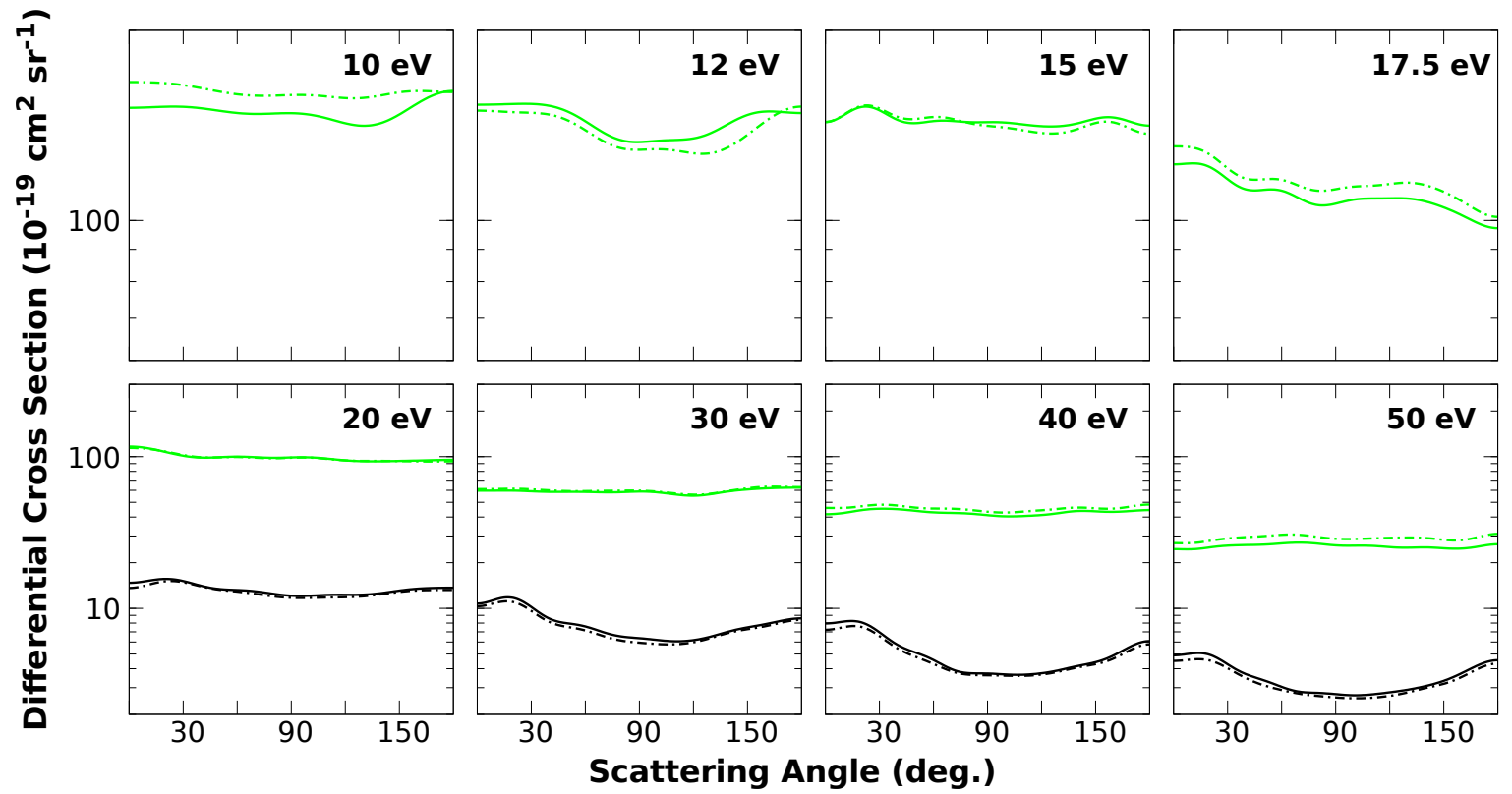

FIG. 9. As in Fig. 8, but for the second band (summed contributions of the $\mathrm{T}_{2}, \mathrm{~T}_{3}, \mathrm{~T}_{4}, \mathrm{~S}_{2}, \mathrm{~S}_{3}$ and $\mathrm{S}_{4}$ states).

et $a .^{56}$ (data at $17.5 \mathrm{eV}$ was obtained with a cubic interpolation). These are displayed as blue curves in Fig. 10 and represent our best estimate of the actual TCSs of ethanol.

Before comparing the available results, we mention that Fig. 10 contains the experimental data of Schmieder ${ }^{16}$ scaled up by $25 \%$, as discussed by Silva et al. ${ }^{17}$ These authors have recently measured TCSs above $50 \mathrm{eV}$, and argued that the old data of Schmieder ${ }^{16}$ (reported below 50 $\mathrm{eV}$ ) should be scaled up in order to obtain a smooth matching to their results. From the theoretical side, TCSs in this energy range were reported in four studies, all accounting only for the trans conformer. A revised additivity rule calculation ${ }^{18}$ improved upon a previous calculation of this type (not shown $)^{17}$, and slightly overestimates the experimental data. Scattering calculations based on effective potentials ${ }^{19,20}$ provided similar TCSs to each other, but underestimate the experiment. A fair comparison to our results should consider the contribution from the trans conformer only, which was the case in the other reports. Our TCSs are smoother than in the previous calculations, and reproduce more accurately the shape of the experimental curve. Additionally, the magnitudes are closer to measurements when compared to the optical potential results ${ }^{19,20}$. When the contribution from the gauche conformer is accounted for, the TCSs lie below the experiment by $\approx 25$ $a_{0}^{2}$, but the overall shape of the curve remains the same. 


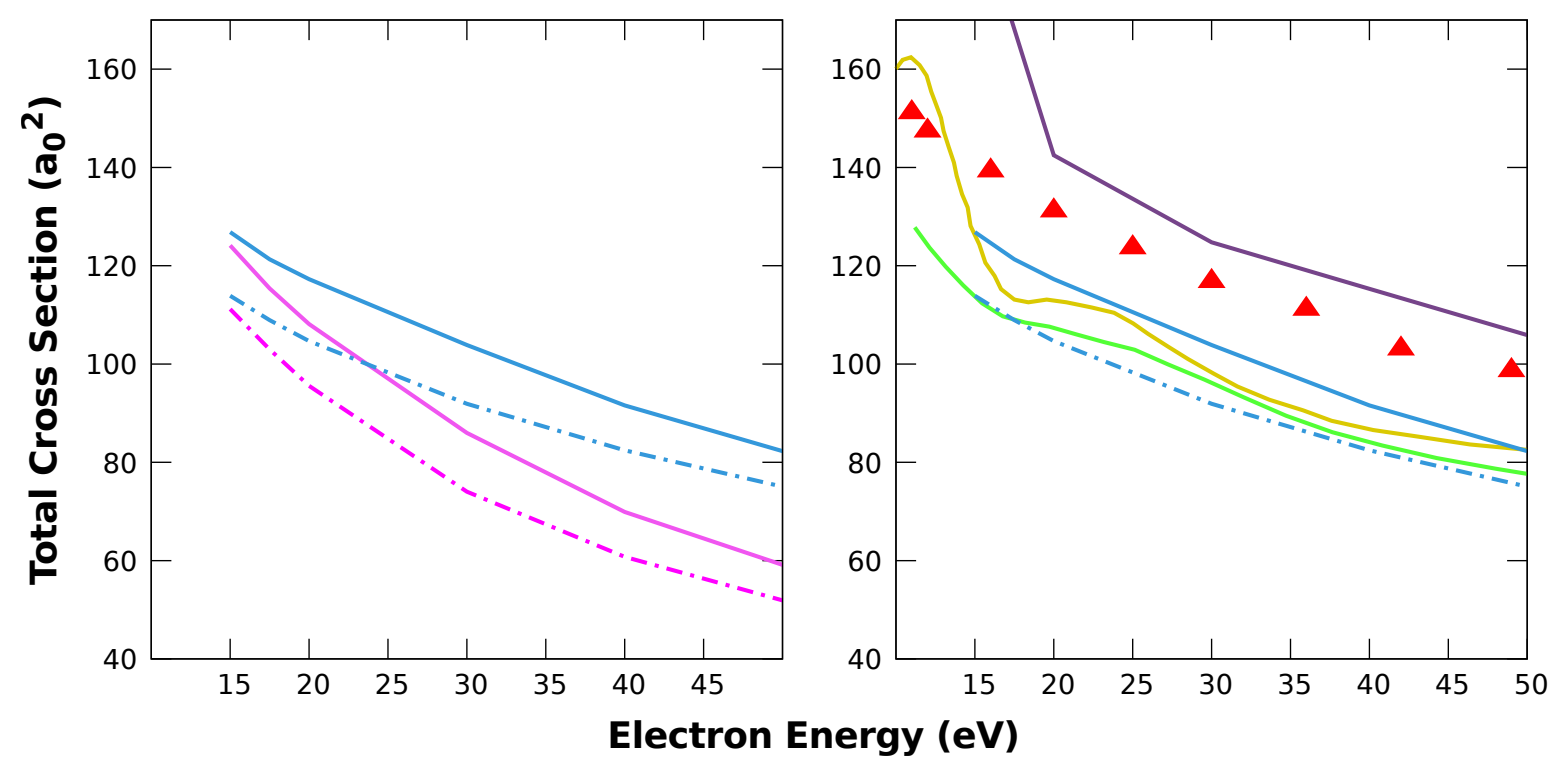

FIG. 10. Total cross sections for electron scattering from ethanol. On the left, our $N_{\text {open }}=183$ SMC results for the trans conformer (full lines) and for the conformational average (dot-dashed lines), with (blue) or without (magenta) the contribution from the measured ionization cross sections ${ }^{56}$. On the right, comparison among results obtained with the present SMC calculations (blue), a revised additivity rule ${ }^{18}$ (purple) and optical potential scattering calculations of Ref. 19 (yellow) and Ref. 20 (green). The measured data ${ }^{16}$ is scaled up by $25 \%$ (red triangles), as discussed in Ref. 17.

\section{CONCLUSIONS}

Differential cross sections for electron impact excitation of the lower lying excited states of ethanol have been computed with the SMC method. We compared them to available experimental data for two distinct bands: one comprising $\mathrm{S}_{1}$ and $\mathrm{T}_{1}$ states, and a second one which should have contributions from three singlets and three triplets. Excitation energies obtained with EOMCCSD calculations support this number of states for the second band, which differs from previous assignments. We have developed a new protocol for selecting the most important single excitations for describing the target states below a given cutoff energy. Making use of it and counting with the recent improvements in our computational implementation of the SMC method, we were able to account for 431 open channels in the scattering calculations.

Concerning the elastic channel, we found that accounting only for the trans tautomer would misleadingly suggest a rather good agreement with the experimental data. However, when the gauche tautomer is considered, as it should, the comparison becomes less satisfactory. The same 
behaviour has been observed for the TCSs, which were compared to previous theoretical and experimental results in the $15-50 \mathrm{eV}$ energy range. At the higher impact energies, the computed excitation DCSs agree reasonably well with available measurements, in shape and magnitude, for scattering angles above $\approx 30^{\circ}$. The relative intensities of each band could also be described. While the measured forward peak was qualitatively reproduced with a simple FBA calculation, the Born-corrected SMC calculation did not, which means the lower partial waves of the latter deserve further improvement. The disagreement we have seen at $10 \mathrm{eV}$ is more intriguing. We notice that just below the ionization potential there is an infinite number of Rydberg states that can play a role in the flux competition, and accounting for a significant part of these states would require the use of more diffuse basis sets.

In some cases (in the elastic channel most noticeably), increasing the number of open channels in the calculations resulted in DCSs somewhat below what has been measured. Thus, obtaining converged cross sections requires more than simply allowing more excited states to compete for the flux. Improving on the accuracy of their description and better accounting for the long range interaction should also affect the underlying multichannel couplings. Given the current capabilities of the SMC code in dealing only with single excitations, the presently proposed procedure for describing the excited states (the TCIS prescription, based on the FCIS coefficients) represents an important improvement in this direction, in comparison to previous MOB-SCI calculations. It provides more accurate excited states, and allows for many more open channels to be accounted for.

The present work illustrates that multichannel calculations with hundreds of open channels are now computationally feasible for molecules of the size of ethanol. We plan to revisit smaller systems like $\mathrm{H}_{2} \mathrm{O}$ and $\mathrm{C}_{2} \mathrm{H}_{4}$ to exploit the new procedures presented here at the limit of our current computational implementation of the SMC method.

\section{ACKNOWLEDGMENTS}

A.G.F. and M.A.P.L. acknowledge support from the Brazilian agency Conselho Nacional de Desenvolvimento Científico e Tecnológico $(\mathrm{CNPq})$. This research used the computing resources and assistance of the John David Rogers Computing Center (CCJDR) in the Institute of Physics “Gleb Wataghin”, University of Campinas. F.K. and M.A.P.L. acknowledge funding from the São Paulo Research Foundation (FAPESP) under Grant No. 2015/23792-5. F.K. also acknowledges 
funding from the WSPLIT project (No. ANR-17-CE05-0005-01).

\section{DATA AVAILABILITY STATEMENT}

The data that support the findings of this study are available from the corresponding author upon reasonable request.

\section{REFERENCES}

${ }^{1}$ U. Nations., Sustainable Development Goals Report 2016 (UN, 2016).

${ }^{2}$ M. O. Dias, T. L. Junqueira, O. Cavalett, M. P. Cunha, C. D. Jesus, C. E. Rossell, R. M. Filho, and A. Bonomi, Bioresour. Technol. 103, 152 (2012).

${ }^{3}$ J. Amorim, C. Oliveira, J. A. Souza-Corrêa, and M. A. Ridenti, Plasma Process. Polym. 10, 670 (2013).

${ }^{4}$ J. Loureiro and J. Amorim, Kinetics and Spectroscopy of Low Temperature Plasmas (2016).

${ }^{5}$ N. Spyrou and J. d. Amorim, IEEE Trans. Plasma Sci. 47, 1583 (2019).

${ }^{6}$ E. M. de Oliveira, S. d'A. Sanchez, M. H. F. Bettega, A. P. P. Natalense, M. A. P. Lima, and M. T. d. N. Varella, Phys. Rev. A 86, 020701 (2012).

${ }^{7}$ Y. Sun and J. Cheng, Bioresour. Technol. 83, 1 (2002), reviews Issue.

${ }^{8}$ M. A. Ridenti, J. A. Filho, M. J. Brunger, R. F. da Costa, M. T. d. N. Varella, M. H. Bettega, and M. A. Lima, Eur. Phys. J. D 70, 161 (2016).

${ }^{9}$ M. J. Brunger, Int. Rev. Phys. Chem. 36, 333 (2017).

${ }^{10}$ Lopes, M. Cristina A., Pires, Wesley A. D., Nixon, Kate L., Amorim, Raony A. A., da Silva, Daniel G. M., Fernandes, Anne C. P., Ghosh, Santunu, Jones, Darryl B., Campbell, Laurence, Neves, Rafael F. C., Duque, Humberto V., García, Gustavo, Blanco, Francisco, and Brunger, Michael J., Eur. Phys. J. D 74, 88 (2020).

${ }^{11}$ M. A. Khakoo, J. Blumer, K. Keane, C. Campbell, H. Silva, M. C. A. Lopes, C. Winstead, V. McKoy, R. F. da Costa, L. G. Ferreira, M. A. P. Lima, and M. H. F. Bettega, Phys. Rev. A 77, 042705 (2008).

${ }^{12}$ M. Fujimoto, W. Brigg, and J. Tennyson, Eur. Phys. J. D 66, 204 (2012).

${ }^{13}$ V. S. Prabhudesai, A. H. Kelkar, D. Nandi, and E. Krishnakumar, Phys. Rev. Lett. 95, 143202 (2005). 
${ }^{14}$ M. Orzol, I. Martin, J. Kocisek, I. Dabkowska, J. Langer, and E. Illenberger, Phys. Chem. Chem. Phys. 9, 3424 (2007).

${ }^{15}$ B. C. Ibănescu, O. May, A. Monney, and M. Allan, Phys. Chem. Chem. Phys. 9, 3163 (2007).

${ }^{16}$ F. Schmieder, Zeitschrift für Elektrochemie und angewandte physikalische Chemie 36, 700 (1930).

${ }^{17}$ D. G. M. Silva, T. Tejo, J. Muse, D. Romero, M. A. Khakoo, and M. C. A. Lopes, J. Phys. B: At., Mol. Opt. Phys. 43, 015201 (2009).

${ }^{18}$ X.-M. Tan and D.-H. Wang, Nucl. Instrum. Methods Phys. Res. B 269, 1094 (2011).

${ }^{19}$ M.-T. Lee, G. L. C. de Souza, L. E. Machado, L. M. Brescansin, A. S. dos Santos, R. R. Lucchese, R. T. Sugohara, M. G. P. Homem, I. P. Sanches, and I. Iga, J. Chem. Phys. 136, 114311 (2012).

${ }^{20}$ A. Bharadvaja, S. Kaur, and K. L. Baluja, Eur. Phys. J. D 73, 251 (2019).

${ }^{21}$ K. Sunanda, A. K. Das, and B. Rajasekhar, J. Quant. Spectrosc. Radiat. Transf. 237, 106609 (2019).

${ }^{22}$ L. R. Hargreaves, M. A. Khakoo, C. Winstead, and V. McKoy, J. Phys. B: At., Mol. Opt. Phys. 49, 185201 (2016).

${ }^{23}$ K. Takatsuka and V. McKoy, Phys. Rev. A 24, 2473 (1981).

${ }^{24}$ K. Takatsuka and V. McKoy, Phys. Rev. A 30, 1734 (1984).

${ }^{25}$ M. A. P. Lima, L. M. Brescansin, A. J. R. da Silva, C. Winstead, and V. McKoy, Phys. Rev. A 41, 327 (1990).

${ }^{26}$ R. F. da Costa, M. T. d. N. Varella, M. H. F. Bettega, and M. A. P. Lima, Eur. Phys. J. D 69, 159 (2015).

${ }^{27}$ M. H. F. Bettega, L. G. Ferreira, and M. A. P. Lima, Phys. Rev. A 47, 1111 (1993).

${ }^{28}$ R. F. da Costa, F. J. da Paixão, and M. A. P. Lima, J. Phys. B: At., Mol. Opt. Phys. 38, 4363 (2005).

${ }^{29}$ sWilliam J. Hunt and W. A. Goddard, Chem. Phys. Lett. 3, 414 (1969).

${ }^{30}$ R. F. da Costa, M. H. F. Bettega, M. T. d. N. Varella, E. M. de Oliveira, and M. A. P. Lima, Phys. Rev. A 90, 052707 (2014).

${ }^{31}$ R. F. da Costa, M. H. F. Bettega, M. A. P. Lima, M. C. A. Lopes, L. R. Hargreaves, G. Serna, and M. A. Khakoo, Phys. Rev. A 85, 062706 (2012).

${ }^{32}$ R. F. da Costa, E. M. de Oliveira, M. H. F. Bettega, M. T. d. N. Varella, D. B. Jones, M. J. Brunger, F. Blanco, R. Colmenares, P. Limão-Vieira, G. García, and M. A. P. Lima, J. Chem. 
Phys. 142, 104304 (2015).

${ }^{33}$ D. B. Jones, F. Blanco, G. García, R. F. da Costa, F. Kossoski, M. T. d. N. Varella, M. H. F. Bettega, M. A. P. Lima, R. D. White, and M. J. Brunger, J. Chem. Phys. 147, 244304 (2017).

${ }^{34}$ D. B. Jones, R. F. da Costa, F. Kossoski, M. T. d. N. Varella, M. H. F. Bettega, F. Ferreira da Silva, P. Limão-Vieira, G. García, M. A. P. Lima, R. D. White, and M. J. Brunger, J. Chem. Phys. 148, 124312 (2018).

${ }^{35}$ R. F. da Costa, J. C. Ruivo, F. Kossoski, M. T. d. N. Varella, M. H. F. Bettega, D. B. Jones, M. J. Brunger, and M. A. P. Lima, J. Chem. Phys. 149, 174308 (2018).

${ }^{36}$ R. F. da Costa, M. T. do N. Varella, M. H. F. Bettega, R. F. C. Neves, M. C. A. Lopes, F. Blanco, G. García, D. B. Jones, M. J. Brunger, and M. A. P. Lima, J. Chem. Phys. 144, 124310 (2016).

${ }^{37}$ D. B. Jones, R. F. C. Neves, M. C. A. Lopes, R. F. da Costa, M. T. do N. Varella, M. H. F. Bettega, M. A. P. Lima, G. García, P. Limão-Vieira, and M. J. Brunger, J. Chem. Phys. 144, 124309 (2016).

${ }^{38}$ G. M. Moreira, F. Kossoski, M. H. F. Bettega, and R. F. da Costa, J. Phys. B: At., Mol. Opt. Phys. 53, 085002 (2020).

${ }^{39}$ J. R. Durig, H. Deeb, I. D. Darkhalil, J. J. Klaassen, T. K. Gounev, and A. Ganguly, J. Mol. Struct. 985, 202 (2011).

${ }^{40}$ E. A. Gardner, A. Nevarez, M. Garbalena, and W. C. Herndon, J. Mol. Struct. 784, 249 (2006).

${ }^{41}$ Y. Tang, X. Shan, S. Niu, Z. Liu, E. Wang, N. Watanabe, M. Yamazaki, M. Takahashi, and X. Chen, J. Phys. Chem. A 121, 277 (2017).

${ }^{42}$ C. Møller and M. S. Plesset, Phys. Rev. 46, 618 (1934).

${ }^{43}$ R. A. Kendall, T. H. Dunning, and R. J. Harrison, J. Chem. Phys. 96, 6796 (1992).

${ }^{44}$ G. B. Bachelet, D. R. Hamann, and M. Schlüter, Phys. Rev. B 26, 4199 (1982).

${ }^{45}$ M. H. F. Bettega, A. P. P. Natalense, M. A. P. Lima, and L. G. Ferreira, Int. J. Quantum Chem. 60, 821 (1996).

${ }^{46}$ T. N. Rescigno and B. I. Schneider, Phys. Rev. A 45, 2894 (1992).

${ }^{47}$ K. Emrich, Nucl. Phys. A 351, 379 (1981).

${ }^{48}$ H. Sekino and R. J. Bartlett, Int. J. Quantum Chem. 26, 255 (1984).

${ }^{49}$ R. J. Bartlett, WIREs Comput. Mol. Sci. 2, 126 (2012).

${ }^{50}$ M. J. Frisch, G. W. Trucks, H. B. Schlegel, G. E. Scuseria, M. A. Robb, J. R. Cheeseman, G. Scalmani, V. Barone, B. Mennucci, G. A. Petersson, H. Nakatsuji, M. Caricato, X. Li, H. P. Hratchian, A. F. Izmaylov, J. Bloino, G. Zheng, J. L. Sonnenberg, M. Hada, M. Ehara, K. Toyota, 
R. Fukuda, J. Hasegawa, M. Ishida, T. Nakajima, Y. Honda, O. Kitao, H. Nakai, T. Vreven, J. A. Montgomery, Jr., J. E. Peralta, F. Ogliaro, M. Bearpark, J. J. Heyd, E. Brothers, K. N. Kudin, V. N. Staroverov, R. Kobayashi, J. Normand, K. Raghavachari, A. Rendell, J. C. Burant, S. S. Iyengar, J. Tomasi, M. Cossi, N. Rega, J. M. Millam, M. Klene, J. E. Knox, J. B. Cross, V. Bakken, C. Adamo, J. Jaramillo, R. Gomperts, R. E. Stratmann, O. Yazyev, A. J. Austin, R. Cammi, C. Pomelli, J. W. Ochterski, R. L. Martin, K. Morokuma, V. G. Zakrzewski, G. A. Voth, P. Salvador, J. J. Dannenberg, S. Dapprich, A. D. Daniels, Ö. Farkas, J. B. Foresman, J. V. Ortiz, J. Cioslowski, and D. J. Fox, “Gaussian 09 Revision D.01,” Gaussian Inc. Wallingford CT 2009.

${ }^{51}$ D. Salahub and C. Sandorfy, Chem. Phys. Lett. 8, 71 (1971).

${ }^{52}$ M. Robin and N. Kuebler, J Electron Spectros. Relat. Phenomena 1, 13 (1972).

${ }^{53}$ W.-C. Tam and C. Brion, J Electron Spectros. Relat. Phenomena 3, 263 (1974).

${ }^{54}$ R. Feng and C. Brion, Chem. Phys. 282, 419 (2002).

${ }^{55}$ K. Varela, L. R. Hargreaves, K. Ralphs, M. A. Khakoo, C. Winstead, V. McKoy, T. N. Rescigno, and A. E. Orel, J. Phys. B: At., Mol. Opt. Phys. 48, 115208 (2015).

${ }^{56}$ K. Nixon, W. Pires, R. Neves, H. Duque, D. Jones, M. Brunger, and M. Lopes, International Journal of Mass Spectrometry 404, 48 (2016). 